\title{
Tenir compte de l'évaluation dans la prise de décision de politiques de développement: le cheminement de l'Association camerounaise de l'évaluation du développement
}

\author{
Author: \\ Debazou Y. Yantio \\ Affiliation: \\ ${ }^{1}$ Cameroon Association of \\ Development Evaluation, \\ Cameroon \\ Correspondence to: \\ Debazou Yantio \\ Email: \\ yantio@hotmail.com \\ Postal address: \\ B.P. 30073 Yaoundé, \\ Cameroon \\ Dates: \\ Received: 21 Aug. 2013 \\ Accepted: 09 Sept. 2013 \\ Published: 01 Nov. 2013 \\ How to cite this article: \\ Yantio, D.Y., 2013, 'Tenir \\ compte de l'évaluation \\ dans la prise de décision de \\ politiques de développement: \\ le cheminement de \\ l'Association camerounaise \\ de l'évaluation du \\ développement', African \\ Evaluation Journal 1(1), Art. \\ \#47, 4 pages. http://dx.doi. \\ org/10.4102/aej.v1i1.47

\section{Copyright:} \\ (C) 2013. The Authors. \\ Licensee: AOSIS \\ OpenJournals. This work \\ is licensed under the \\ Creative Commons \\ Attribution License.
}

Read online:

\section{Introduction}

D'après Kosheleva et Segone (2013:9), le développement des organisations volontaires nationales de l'évaluation professionnelle (VOPE) a débuté à la fin des années 1970 et au début des années 1980. Le nombre de VOPE nationales et régionales a augmenté pour passer de 19 dans les années 1990 à 171 en mars 2013 (Rugh 2013:15-16). En Afrique, l'Association africaine de l'évaluation (AfrEA) a été créée en 1999. Au cours des 15 dernières années, plusieurs associations nationales ont été mises en place et se battent en vue de leur institutionnalisation. Bien que toutes ces associations relèvent de contextes nationaux et culturels uniques, elles partagent des buts et défis communs. Cet article décrit le contexte de la genèse et du développement de la CaDEA (Association camerounaise de l'évaluation du développement) afin de partager avec les autres associations nationales les avancées et résultats obtenus, les obstacles et défis auxquels la CaDEA est confronté, les facteurs clés qui ont influencé ce cheminement spécifique, les prochaines étapes, les innovations organisationnelles qui ont été imaginées et les enseignements tirés de cette expérience, dans le but de contribuer à déterminer comment placer l'évaluation au centre des processus de prise de décisions relatives au développement et assurer que les efforts des pays et des partenaires gagnent en efficacité.

\section{Contexte et genèse}

Le Cameroun est un pays en développement situé en Afrique centrale, au dessus de l'équateur, au fond du golfe de Guinée, à la limite de l'Afrique occidentale. Il fait frontière avec le Nigeria à l'Ouest, le Tchad, la République centrafricaine et le Congo à l'Est, et le Gabon et la Guinée équatoriale au Sud. Par la diversité de ses paysages, de ses peuples et de ses cultures, le Cameroun est une " Afrique en miniature ». Le pays compte plus de 255 ethnies et autant de langues locales, mais deux langues officielles ont été adoptées: le français et l'anglais. Le pays est dirigé par un président de la République qui incarne l'exécutif, avec un parlement bicaméral chargé d'élaborer les lois et d'évaluer l'action gouvernementale. Le Sénat, qui est venu compléter l'Assemblée nationale déjà existante depuis l'indépendance en 1960, a été institué en 1996; il est devenu fonctionnel en mai 2013. Le pouvoir judiciaire est représenté par la Cour suprême et les juridictions subsidiaires.

L'idée de créer l'Association camerounaise de l'évaluation du développement (CaDEA) s'est imposée après la participation de son fondateur au Programme international de formation à l'évaluation du développement (IPDET) en juillet 2003 à l'Université de Carleton à Ottawa, au Canada. Comme pour les autres associations affiliées à l'AfrEA (Kriel 2006), sa raison d'être était « de sensibiliser et accroître la demande d'évaluation, et bâtir une communauté de parties concernées par l'évaluation [dans le pays], mais aussi de faciliter l'accès des professionnels camerounais de l'évaluation aux opportunités de formation offertes à travers le monde en vue du renforcement de leurs capacités techniques ». Un problème fondamental devait être résolu avant la mise sur pied du présent projet: comment informer et rallier les professionnels de l'évaluation travaillant au Cameroun et dans le monde entier? Lise Kriel, alors secrétaire de l'Association africaine d'évaluation et collaboratrice de la deuxième présidente de l'association, Zenda Ofir, conseilla d'établir un gestionnaire de liste de diffusion, ce qui fût fait chez www.topica.com. C'est ainsi que 1'adresse cadea@topica.com fut créée en décembre 2003 par le fondateur de la CaDEA; ce dernier est l'actuel « propriétaire » de cette liste, qui présente des difficultés de gestion liées à l'évolution de l'entreprise qui entretient ce service de messagerie de groupe. Afin de bénéficier des autres options en matière de gestion de liste de diffusion, evaluationcameroon@yahoogroups. com fut créée le 17 juin 2006; cette dernière est dotée de fonctionnalités de gestion complètes et innovantes. 
La première réunion en face-à-face de la CaDEA s'est tenue le 17 janvier 2007 lors de la $3^{e}$ Conférence internationale biennale de l'AfrEA à Niamey au Niger (de manière surprenante, hors du Cameroun, le pays d'origine). La célèbre salle SAYO, lieu chargé d'histoire de l'Hôtel Terminus accueillit cette importante rencontre préparatoire au lancement officiel des activités de la CaDEA. Six évaluateurs ont pris part à cette première réunion en face-à-face. Seuls cinq d'entre eux restèrent jusqu'à la fin de la réunion. Ce groupe restreint de membres fondateurs a par la suite organisé des réunions à leurs domiciles à Douala et à Yaoundé, au Cameroun. Ces évaluateurs se sont déplacés de leurs villes respectives pour Niamey, témoignant de leur ferme volonté de participer et de porter l'association sur les fonts baptismaux.

\section{Les avancées et résultats obtenus}

En plus des listes électroniques suscitées qui permettent de regrouper les évaluateurs, nouveaux et chevronnés, intéressés par l'état de l'évaluation du développement au Cameroun, il est aujourd'hui possible de consulter les pages de la CaDEA sur Internet à l'adresse www.cadea.org, assurant la présence effective de l'association en ligne et permettant aux membres potentiels et autres personnes concernées d'obtenir des informations par courrier électronique en écrivant directement à info@cadea.org.

Aujourd'hui, la CaDEA compte 62 membres Camerounais et non-Camerounais, résidant au Cameroun et à l'étranger, dont 32 membres en règle. Les femmes comptent pour $25 \%$ de l'effectif total. Plus important, la CaDEA a tenu son Assemblée générale constituante le 5 mai 2012, à laquelle 18 membres ont assisté. On notera notamment trois accomplissements majeurs à l'issue de cette Assemblée: (i) l'adoption des statuts; (ii) l'élection d'un bureau composé de quatre membres (président, vice-président, secrétaire général, trésorier); et (iii) l'adoption d'un cadre stratégique à moyen terme (2012-2014).

Dans l'optique de faire de l'organisation une entité viable dotée des capacités nécessaires pour mener à bien sa mission, les priorités stratégiques pour 2012-2014 ont été définies ainsi: attirer de nouveaux membres, susciter davantage de motivation chez les membres pour en faire la force motrice de l'Association, développer des partenariats et assurer une gestion professionnelle de l'Association. Dès lors que les activités de l'Association prendront de l'ampleur et afin de suppléer l'indisponibilité des membres du bureau lorsqu'ils sont pris par leurs occupations professionnelles, il est prévu de recruter un directeur chargé de gérer les affaires au quotidien, sous la supervision du bureau de l'Association. En ce qui concerne le partenariat, d'autres associations d'évaluation ont été contactées afin de discuter des plans d'adoption et d'adaptation de leurs procédés et de leurs logiciels pertinents pour les opérations de la CaDEA. À mesure que les activités de l'Association se développent, il est prévu de procéder au recrutement d'un directeur chargé de gérer les affaires au quotidien, sous la supervision d'un bureau de l'Association. Ce modèle d'organisation reste approprié car les membres du bureau sont bénévoles et ne sont souvent pas disponibles à tout moment pour mener à bien les activités pour lesquelles l'Association s'est engagée. Grâce au partenariat et à une gestion professionnelle, la CaDEA, qui est une jeune association, pourra renforcer ses capacités organisationnelles. À propos de ces dernières, nous nous inspirons davantage de Kriel (2006) que des récents auteurs publiant sur le renforcement des capacités en matière d'évaluation: « les capacités organisationnelles englobent le leadership, les membres, les services, les finances, les ressources humaines, les liens inter-organisationnels et les structures organisationnelles globales qui contribuent à regrouper toutes ces ressources ». En effet, cette approche vise les trois niveaux d'action qui sont (i) un environnement propice à l'évaluation (niveau structurel); (ii) le niveau individuel; et (iii) le niveau institutionnel (Heider, 2011).

En plus du nombre croissant de membres, le sentiment d'appartenance à l'Association et la ferme volonté de bâtir un brillant avenir pour l'évaluation au Cameroun constituent un capital (social) qui sera nécessaire pour faire avancer l'agenda de la promotion de l'évaluation.

Il convient également de noter que, chose surprenante, la CaDEA est de plus en plus connue au niveau de la communauté internationale de l'évaluation comme un instrument fiable de promotion de l'évaluation et un fervent partisan d'une efficacité accrue des politiques et programmes en Afrique et ailleurs. En effet, la CaDEA et ses membres participent activement aux initiatives internationales et leurs propositions suscitent l'intérêt et du soutien. Pour s'aligner sur la communauté internationale de l'évaluation du développement, la CaDEA s'est affiliée à l'AfrEA et à l'Organisation internationale de coopération en évaluation (IOCE) en 2004 et 2006 respectivement. Par ailleurs, en septembre 2004, la CaDEA a été représentée au siège de l'Organisation internationale de la francophonie (OIF) à Paris, en France, lors de la première réunion constituante du Réseau francophone de l'évaluation (RFE), le réseau des évaluateurs francophones.

\section{Goulots d'étranglement et défis}

Autour de la question savoir si l'Association devrait ou non offrir des services de consultants, les membres de la CaDEA se sont trouvés confrontés à trois principaux défis: (i) le fragile consensus sur la nature et l'objectif de l'association; (ii) la compréhension diverse de la situation de l'évaluation, de la situation de l'offre et de la demande d'évaluation dans le pays et dans le monde entier; et (iii) l'engagement insuffisant des membres. Parvenir à un consensus sur la question n'a pas été aisé. Mais après un débat laborieux, nous avons décidé d'en faire une organisation professionnelle, à but non lucratif, suivant en cela le conseil tiré de l'expérience des associations qui ont réussi et de celles qui ont échoué parmi celles qui étaient affiliés à l'AfrEA (Kriel 2006). Cette option qui a été retenue évite à l'Association de se retrouver en situation de conflit d'intérêt avec ses membres, qui sont pour la plupart des consultants offrant des services payants 
en tant qu'individus ou cabinets-conseils. En outre, il s'agit pour l'Association de rester indépendant, loin des intérêts particuliers, afin de travailler pour l'intérêt public.

La différence d'expérience en évaluation entre les membres n'a pas facilité la prise de décisions, y compris l'expérience relative à l'élaboration d'un plan stratégique. La CaDEA regroupe des divers professionnels: des étudiants en cours de formation qui souhaitent faire carrière dans l'évaluation; des évaluateurs novices qui ont peu ou pas d'expérience pratique de l'évaluation; des professionnels chevronnés de l'évaluation; des commanditaires de l'évaluation, etc. Ces groupes de personnes disposent de niveaux de connaissances techniques en évaluation du développement variés, en particulier des normes et standards applicables. Cette diversité se traduit par une compréhension différente de ce qu'est l'évaluation du développement, les facteurs d'influence, la finalité et les buts de la promotion de l'évaluation et son utilité dans un pays comme le Cameroun. Dans de telles conditions, dégager pour l'Association une stratégie et un plan de travail réaliste et pertinent s'est avéré un exercice difficile. Afin de limiter les dissensions et converger rapidement vers un consensus, une stratégie de trois ans a été adoptée, traitant seulement des priorités les plus urgentes, abandonnant les actions stratégiques qui séparaient profondément les membres au cours de la discussion lors de l'Assemblée générale constitutive. On espère que la stratégie sera étoffée au fur et à mesure du renforcement des capacités techniques des membres et que l'Association se consolide.

Par ailleurs, l'établissement d'un plan de travail et l'acceptation des tâches de mise en application ont été compromis par l'incompatibilité des emplois du temps des membres. Ceux-ci ne trouvaient pas assez de temps pour répondre aux attentes du groupe.

\section{Facteurs favorables clés}

Si l'Association existe grâce au dynamisme de ses fondateurs, à l'engagement important de quelques personnes dévouées, son développement résulte quant à lui de l'importance croissante de l'évaluation et de la gestion axée sur les résultats (GAR) dans le pays. En effet, cet élan vient non seulement de la demande sociale de la population qui, de plus en plus, exige des résultats du gouvernement, mais aussi de l'exigence des bailleurs de fonds qui attendent des dirigeants qu'ils rendent compte (Rist, Boily \& Martin, 2011). Ceci est vrai pour le Cameroun, mais j'estime que le dernier facteur est prépondérant. La création de la liste de diffusion électronique de la CaDEA a également joué un rôle primordial dans le développement du réseau national et le renforcement des liens entre les membres.

\section{Prochaines étapes}

La CaDEA entend aller au-delà des principales activités de partage d'expériences, de soutien mutuel et d'assistance de la part des organisations d'évaluateurs listées par Quesnel (2006) et tenir un rôle plus actif dans la promotion et l'intégration de l'évaluation dans la prise de décision des politiques et programmes de développement au Cameroun. Les objectifs stratégiques pour la période 2012-2014 sont les suivants:

(i) augmenter le nombre des membres pour passer de 50 en décembre 2012 à 150 à la fin de l'année 2014;

(ii) développer des partenariats stratégiques à l'échelle nationale et internationale pour (a) renforcer les capacités techniques des membres; (b) accroître la visibilité de l'association, et (c) placer les membres à des postes vacants liés à l'évaluation;

(iii) s'agissant du plaidoyer, (a) au moins $25 \%$ des programmes et projets dans l'administration prévoient ou effectuent l'évaluation de programme; (b) sensibiliser les décideurs et les chefs de programmes sur la nature et les avantages de l'évaluation; (c) recueillir et disséminer les informations sur les pratiques concernant le suivi et l'évaluation dans le pays.

\section{Innovations et enseignements tirés}

Trois enseignements sont à tirer: (i) susciter davantage la motivation du petit nombre d'individus qui demeurent actifs au sein de l'Association et qui, plus que les autres, ne ménagent ni leur temps, ni leurs ressources, (ii) rester innovant, et (iii) privilégier l'analyse structurée et rigoureuse de la situation en place afin d'élaborer une stratégie pour répondre aux problèmes et défis et saisir les opportunités existantes et à venir pour améliorer le statut et l'utilité de l'évaluation de programmes et politiques dans le pays.

Le cheminement de la CaDEA est conforme au « scenario typique de la formation des réseaux des évaluateurs » de Rugh (2013:18) qui observe que « quelques individus dépassent leur nature compétitive et décident de se mettre ensemble de manière informelle pour partager les expériences concernant les méthodes appliqués à l'évaluation ». Dans un tel contexte, la motivation continue et renouvelée du fondateur et du noyau initial des membres a été déterminante pour la survie du réseau informel au fil du temps jusqu'à sa formalisation en 2012. Ces conditions continuent d'ailleurs d'être pertinentes avec des membres qui sont tiraillés par leurs occupations professionnelles, car il ne faut pas l'oublier, dans la majorité des cas, chaque membre a d'abord une occupation principale qui lui permet de gagner sa vie; ils contribuent à la vie de l'association de manière bénévole en mettant selon leur volonté leur temps à la disposition du groupe. C'est d'abord à ce niveau que les dirigeants de l'Association doivent être inventifs.

En effet, l'innovation sert à trouver des solutions simples, peu coûteuses et adaptées au contexte des problèmes et défis auxquels fait face l'Association dans la conduite de ses affaires. Il en est ainsi du problème de la motivation continue des membres évoqué ci-dessus. En effet, comment convaincre les membres de consacrer assez de leur temps et engager parfois leurs propres ressources pour effectuer les activités de l'association? Parmi les innovations, on notera des incitations particulières, comme la valorisation des contributions 
spécifiques des membres, et le partage des bénéfices non financiers tirés du fonctionnement de 1'Association. Lorsqu'un membre effectue une activité majeure, cette contribution est enregistrée et largement diffusée parmi les membres, en insistant sur l'exemple à suivre. En outre, les membres qui contribuent plus souvent se voient confier des responsabilités plus valorisantes au sein de l'organisation (par exemple chef de projet ou point focal d'un partenaire institutionnel) ou sont envoyés comme représentants de l'Association pour participer à des évènements dans le pays ou à l'étranger. En outre, les membres disposent d'un accès gratuit à certains services de l'Association tels que les avis d'appel à candidatures et à propositions pour les consultants, etc.). Cette dernière mesure sert à maintenir le sentiment d'appartenance à la communauté et susciter un intérêt minimal pour les activités de l'Association.

Pour augmenter le statut et l'utilité de l'évaluation de programmes dans la prise de décisions de développement dans le pays, les partenaires internationaux, dont les fondations et agences multilatérales de développement (banques de développement, agences des Nations unies), pourraient contribuer en facilitant la réalisation de leurs objectifs habituels, notamment de " contribuer à l'amélioration des connaissances » et «promouvoir la culture de l'évaluation dans (...) les pays ». Cette contribution pourrait être mise en œuvre, non seulement à travers la coopération avec les organisations nationales des professionnels de l'évaluation mais aussi par des soutiens multiformes de la part de ces dernières. Les modalités de la coopération pourraient être les suivantes:

1. Impliquer les organisations nationales des professionnels de l'évaluation dans le processus d'évaluation des pairs lors des évaluations au niveau national.

2. Apporter un soutien financier direct aux initiatives de formation des organisations régionales et nationales des professionnels de l'évaluation.

3. Organiser des rencontres pour partager les connaissances et des évènements médiatiques avec les partenaires nationaux (décideurs, députés, maires, etc.) pour promouvoir la culture de l'évaluation et la prise de décision des politiques de développement basées sur les preuves.

4. Diffuser les produits de l'évaluation et autres connaissances de l'association au niveau national avec la participation active des organisations nationales des professionnels de l'évaluation.
5. Offrir plus d'opportunités de stage aux professionnels et étudiants africains de l'évaluation au niveau du siège et des bureaux pays.

\section{Remerciements}

L'idée de cet article émane de Jim Rugh de l'Organisation internationale pour la coopération en évaluation (IOCE), dans le cadre de l'initiative EvalPartners qui entre autres contribue à la constitution de la base des connaissances nécessaire pour orienter les efforts de développement organisationnel des nouvelles associations nationales voire de celles qui existent depuis plus longtemps. Je lui exprime ma gratitude pour avoir créé l'occasion de cette réflexion et contribution. Je remercie sincèrement Prof. Brad Cousins de l'Université d'Ottawa qui a revu de fond en comble le manuscrit original. Je suis également redevable aux réviseurs et rédacteurs qui m'ont gratifiés de commentaires et suggestions qui ont été fort utiles dans l'amélioration de la qualité globale de cet article. Cependant, je suis seul responsable de toutes les erreurs et imperfections qui subsisteraient.

\section{Intérêts concurrents}

Les auteurs déclarent qu'ils n'ont aucune relation financière ou personnelle(s) qui ont influencé leur façon inappropriée en écrivant cet article.

\section{Références}

Heider, C., 2011, 'A Conceptual Framework for Developing Evaluation Capacities: Building on Good Practice', in R.C. Rist, M.H. Boily \& F. Martin (eds.), Influencing Change: Building Evaluation Capacity to Strengthen Governance, pp. 85-110, The World Bank, Washington, D.C.

Kosheleva, N. \& Segone M., 2013, 'EvalPartners and the role of Voluntary Organizations for Professional Evaluation in the development of national evaluation capacity' in J. Rugh \& M. Segone (eds.), Voluntary Organizations for Professional Evaluation (VOPEs): Learning from Africa, Americas, Asia, Australasia, Europe and Middle East, pp. 8-12, UNICEF, in partnership with Cooperacion Espanola, Ministry of Foreign Affairs of Finland, UNEG, and UN Women, New York.

Kriel, L., 2006, 'How to Build Evaluation Associations and Networks: Learning from the Pioneers', in M. Segone \& A. Ocampo (eds.), Creating and Developing Evaluation Organizations: Lessons Learned from Africa, Americas, Asia, Australasia, Europe, pp. 161- 187, DESCO \& UNICEF, Peru.

Quesnel, J.S., 2006, 'The Importance of Evaluation Associations and Networks', in M. Segone \& A. Ocampo (eds.), Creating and Developing Evaluation Organizations: Lessons Learned from Africa, Americas, Asia, Australasia, Europe, pp. 13-29, DESCO \& UNICEF, Peru.

Rist, R., Boily, M.H. \& Martin F., 2011, 'Evaluation Capacity Building: A Conceptual Framework', in R. Rist, M.H. Boily \& F. Martin (eds.), Influencing Change: Building Evaluation Capacity to Strengthen Governance, pp. 1-12, The World Bank, Washington, D.C.

Rugh, J., 2013, 'The Growth and Evolving Capacities of VOPEs', in J. Rugh \& M. Segone (eds.), Voluntary Organizations for Professional Evaluation (VOPEs): Learning from Africa, Americas, Asia, Australasia, Europe and Middle East, pp. 13-40, UNICEF, in partnership with Cooperacion Espanola, Ministry of Foreign Affairs of Finland, UNEG, and UN Women, New York. 